\title{
Strong stabilization (via weak stabilization) of hybrid systems with a nonmonotone feedback
}

\author{
Judith Vancostenoble*
}

\begin{abstract}
We recall a previous general result of weak stabilization for an abstract second order evolution equation damped by a nonlinear nonmonotone feedback. We apply it to deduce strong stabilization of hybrid systems without global monotonicity.
\end{abstract}

\section{Introduction}

First we recall a previous result of weak stabilization for an abstract evolution equation damped by a nonlinear and nonmonotone feedback $[12,13]$. More precisely we consider some evolution equations of second order for which strong compactness of trajectories is unknown while weak compactness holds. We give general conditions of weak stabilization. This result extends classical results of strong stabilization obtained under hypotheses insuring strong compactness of trajectories (assuming in particular that the feedback is monotone). See for example $[3,6]$ for strong stabilization of the wave equation with an internal damping and also [2] for a general result for an abstract evolution equation.

Then we prove that this result of weak stabilization can be applied to hybrid systems. This gives a first result of weak stabilization without monotonicity assumption on the feedback. Moreover, if we add an assumption of local monotonicity, then, we easily deduce strong stabilization from weak stabilization. This extends for example an earlier result of [5].

\section{General result of weak stabilization}

\subsection{Abstract framework}

Let $X$ be a locally compact space and $\mu$ be a positive measure such that $\mu(X)<+\infty$. We denote by $K(X)=\mathcal{C}_{c}^{0}(X ; \mathbb{R})$ the space of continuous and compactly supported functions from $X$ into $\mathbb{R}$ and by $H$ the Hilbert space $L^{2}(X, \mu)$. Let $A$ be a linear operator on $H$ with dense domain $D(A)$. We assume that $A$ is self-adjoint, coercive

${ }^{*}$ I.R.M.A.R. Université Rennes I et E.N.S. Cachan, Antenne de Bretagne, Campus de Ker Lann, 35170 Bruz, France. e-mail: vancoste@bretagne.ens-cachan.fr 
and that the resolvent of $A$ is compact. We define $V=D\left(A^{\frac{1}{2}}\right)$ equipped with the scalar product

$$
\forall u, v \in V, \quad(u, v)_{V}=\left(A^{\frac{1}{2}} u, A^{\frac{1}{2}} v\right)_{H}=\langle\tilde{A} u, v\rangle_{V^{\prime}, V}
$$

where $\tilde{A} \in \mathcal{L}\left(V ; V^{\prime}\right)$ is defined by the bilinear form $(., .)_{V}$ and extends $A$. As usual we identify $H$ with its dual. Then $V \hookrightarrow H \hookrightarrow V^{\prime}$ with the following relation : $\forall h \in H, \forall v \in V, \quad\langle h, v\rangle_{V^{\prime}, V}=(h, v)_{H}$. Moreover we assume that $\mathcal{E}=K(X) \cap V$ is dense in $V$.

Let also $a: X \rightarrow \mathbb{R}$ be such that $a \in L^{\infty}(X, \mu)$ and $a(x) \geq 0 \mu$-a.e. $x \in X$ and let $q: \mathbb{R} \rightarrow \mathbb{R}$ be a continuous function. Then we consider the following problem for $\left(u_{0}, v_{0}\right)$ given in $V \times H$ :

$$
\left\{\begin{array}{l}
u_{t t}+\tilde{A} u=-a q\left(u_{t}\right), \quad t \in \mathbb{R}_{+}, \\
\left(u, u_{t}\right) \in V \times H, \\
u(0)=u_{0}, u_{t}(0)=v_{0} .
\end{array}\right.
$$

We define the energy of $u$ by

$$
\forall t \in \mathbb{R}_{+}, \quad E(t)=\frac{1}{2}\left(\|u(t)\|_{V}^{2}+\left\|u_{t}(t)\right\|_{H}^{2}\right) .
$$

A straightforward computation gives

$$
\forall t \in \mathbb{R}_{+}, \quad E^{\prime}(t)=-\int_{X} a q\left(u_{t}\right) u_{t} d x .
$$

\subsection{Wellposedness}

We assume that

$$
\exists m \in \mathbb{R} \mid \lambda \mapsto q(\lambda)+m \lambda \text { is increasing. }
$$

Then applying a theorem of Lipschitz perturbation of a maximal monotone operator (see H. Brézis [1]), we prove that (2.1) is wellposed (see [12] for the proof).

Theorem 1. Assume (2.2).

(i) For all $\left(u_{0}, v_{0}\right) \in V \times V$ such that $A u_{0}+q\left(v_{0}\right) \in H$, there exists a unique strong solution $u\left(t ; u_{0}, v_{0}\right)$ of (2.1) satisfying

$$
\forall t \in \mathbb{R}_{+}, \quad\left(u(t), u_{t}(t)\right) \in V \times V, \quad \text { and } \quad A u(t)+q\left(u_{t}(t)\right) \in H,
$$

and, for all $T>0,\left(u, u_{t}\right) \in W^{1, \infty}((0, T) ; V \times H)$.

(ii) For all $\left(u_{0}, v_{0}\right) \in V \times H$, there exists a unique weak solution $u\left(t ; u_{0}, v_{0}\right)$ of (2.1) satisfying $\left(u, u_{t}\right) \in \mathcal{C}^{0}([0,+\infty[; V \times H)$. 


\subsection{Weak stabilization}

In order to study stabilization, we assume

$$
\forall \lambda \in \mathbb{R}, \quad \lambda q(\lambda) \geq 0,
$$

which guarantees that the energy is decreasing (thus the trajectories are bounded). We also assume that

$$
\forall \lambda>0, \quad q(\lambda)>0 .
$$

Moreover, for asymptotic stabilization, we need the following "uniqueness" property :

$$
\begin{gathered}
\left(\varphi \in V, A \varphi=\omega^{2} \varphi \text { and } a(x) \varphi(x)=0 \mu \text {-a.e. } x \in X\right) \\
\Rightarrow \varphi(x)=0 \quad \mu \text {-a.e. } x \in X .
\end{gathered}
$$

Then we prove

Theorem 2. Assume (2.2)-(2.5). For all $\left(u_{0}, v_{0}\right) \in V \times H$, the solution of (2.1) satisfies

$$
\left(u(t), u_{t}(t)\right) \rightarrow(0,0) \text { weakly in } V \times H \text { as } t \rightarrow+\infty .
$$

Remark. Note that (2.4) and(2.5) are necessary conditions for weak stabilization (see respectively [9] and [13] for counter-examples).

\subsection{Comments}

This result of weak stabilization can be applied for example to wave or plate equations with an internal damping. In the case of a wave equation with an internal damping, it extends a previous result of M. Slemrod [10] who also proved weak stabilization but assuming moreover that $q$ is Lipsch=itz continuous. For examples of application, we refer to $[12,13]$ (in which we give a more general result that also applies to boundary or pointwise dampings). For a complete proof, we refer to [12] or to [14] (in the case of the wave equation). (See also [11] for a proof in the spirit of [10], $b=$ ased on Young measures and see [13] for a proof in the spirit of the monotone case of [2] based on the introduction of a convex l.s.c. function). We also refer to A. Haraux [7] for a recent and slightly different proof based on almost periodic functions and to some extensions of Theorem 2 .

Note also that if we add hypotheses insuring compactness of trajectories, we recover the classical results of strong stabilization as a consequence of Theorem 2 . 


\section{Application to hybrid systems}

\subsection{Example}

Let us consider the example of a cable pinched at one end with a tip mass at the free end (concerning this model, see for example [8]) :

$$
\begin{cases}y_{t t}-y_{x x}=0, & t>0,0<x<1, \\ y(0, t)=0, & t>0, \\ y_{t t}(1, t)+y_{x}(1, t)=-q\left(y_{t}(1, t)\right), & t>0, \\ \left(y(x, 0), y_{t}(x, 0)\right)=\left(y^{0}(x), y^{1}(x)\right), & 0<x<1 .\end{cases}
$$

We define the energy of $y$ by

$$
\forall t \geq 0, \quad E(t)=\frac{1}{2} \int_{0}^{1}\left(y_{x}(x, t)^{2}+y_{t}(x, t)^{2}\right) d x+\frac{1}{2} y_{t}(1, t)^{2} .
$$

For regular solutions, classical computations give, (assuming (2.3)),

$$
\forall t \in \mathbb{R}_{+}, \quad E^{\prime}(t)=-q\left(y_{t}(1, t)\right) y_{t}(1, t) \leq 0 .
$$

\subsection{Description of the method}

Let us briefly describe the method : we set $u=(y, y(1))$ in order to obtain (with an appropriate choice of $X, \mu, \ldots)$ an equation of the form (2.1). Then Theorems 1 and 2 imply wellposedness and weak stability :

$$
\left(u(t), u_{t}(t)\right) \underset{t \rightarrow+\infty}{\rightarrow}(0,0) \text { weakly in } V \times H,
$$

i.e.,

$$
\begin{gathered}
\left(y(t), y_{t}(t)\right) \underset{t \rightarrow+\infty}{\rightarrow}(0,0) \text { in } H^{1}(0,1) \times L^{2}(0,1), \\
\left(y(1, t), y_{t}(1, t)\right)_{t \rightarrow+\infty}^{\rightarrow}(0,0) \text { in } \mathbb{R} \times \mathbb{R} .
\end{gathered}
$$

This gives a first result of weak stabilization with no assumption of monotonicity on the feedback. Note that it implies $y_{t}(1, t) \rightarrow 0$ as $t \rightarrow+\infty$. Then we also assume that $q$ is increasing in a neighbourhood of 0 . For $t$ large enough, $y_{t}(1, t)$ belongs to the domain of monotonicity of $q$. Thus the problem reduces to a question of stabilization with a monotone feedback. Then using the classical theory, we obtain strong stabilization.

\subsection{New formulation}

First, we introduce some notations in order to transform (3.1) into (2.1).

Set $X=] 0,1]$. We denote by $\left.K(X)=\mathcal{C}_{c}^{0}(10,1] ; \mathbb{R}\right)$. For all $\varphi \in K(X)$, we set $\psi=\varphi_{\mid] 0,1[} \in C^{0}(] 0,1[; \mathbb{R})$ and $\eta=\varphi(1) \in \mathbb{R}$. Clearly we have

$$
K(X) \subset \mathcal{C}^{0}(] 0,1[; \mathbb{R}) \times \mathbb{R} .
$$


We define the measure $\mu$ by :

$$
\forall \varphi=(\psi, \eta) \in K(X), \quad\langle\mu, \varphi\rangle=\int_{0}^{1} \psi(x) d x+\eta
$$

Let $H:=L^{2}(X, \mu)$ be the Hilbert space $H=L^{2}(0,1) \times \mathbb{R}$, with the scalar product :

$$
\forall v=(z, \eta), \tilde{v}=(\tilde{z}, \tilde{\eta}) \in H, \quad(v, \tilde{v})_{H}=(z, \tilde{z})_{L^{2}(0,1)}+\eta \tilde{\eta} .
$$

We define the operator $A$ by

$$
\begin{gathered}
D(A)=\left\{u=(y, \xi) \in H^{2}(0,1) \times \mathbb{R}: y(0)=0 \text { and } \xi=y(1)\right\} \\
\forall u=(y, \xi) \in D(A), \quad A u=\left(-y_{x x}, y_{x}(1)\right) .
\end{gathered}
$$

$A$ is linear self-adjoint, coercive from $D(A)$ into $H$, with dense doma= in and compact resolvent. Moreover $V:=D\left(A^{\frac{1}{2}}\right)$ is the space

$$
V=\left\{u=(y, \xi) \in H^{1}(0,1) \times \mathbb{R}: y(0)=0 \text { and } \xi=y(1)\right\}
$$

Note that, $\forall u=(y, \xi) \in D(A), \forall \tilde{u}=(\tilde{y}, \tilde{\xi}) \in V$,

$$
(A u, \tilde{u})_{H}=-\int_{0}^{1} y_{x x} \tilde{y} d x+y_{x}(1) \tilde{\xi} .
$$

Thus $\forall u=(y, \xi), \tilde{u}=(\tilde{y}, \tilde{\xi}) \in V$,

$$
(u, \tilde{u})_{V}=\langle\tilde{A} u, \tilde{u}\rangle_{V^{\prime}, V}=\int_{0}^{1} y_{x} \tilde{y}_{x} d x .
$$

Finally, we define $a: X \rightarrow \mathbb{R}$ by $a(1)=1$ and $a_{\mid] 0,1[} \equiv 0$. Clearly $a \in L^{\infty}(X, \mu)$ and $a \geq 0$ on $X$.

Consider now a regular solution $y$ of $(3.1)$ and let $\xi(t)=y(1, t)$. Then (3.1) becomes

$$
\begin{cases}y_{t t}-y_{x x}=0, & t>0,0<x<1 \\ y(0, t)=0, & t>0, \\ \xi_{t t}+y_{x}(1)=-q\left(\xi_{t}\right), & t>0 \\ \left(y(x, 0), \xi(0), y_{t}(x, 0), \xi_{t}(0)\right)=\left(y^{0}(x), y^{0}(1), y^{1}(x), y^{1}(1)\right) . & \end{cases}
$$

Finally letting $u=(y, \xi),(3.2)$ becomes $(2.1)$.

\subsection{Wellposedness}

First we assume that

$$
\forall A>0, \quad \exists m_{A} \in \mathbb{R} \mid \lambda \mapsto q(\lambda)+m_{A} \lambda \quad \text { is increasing. }
$$


Corollary 1. Assume (3.3).

(i) For all $\left(u_{0}, v_{0}\right) \in D(A) \times V$, there exists a unique strong solution $u=(y, \xi)$ of (3.2) satisfying

$$
\left(u(0), u_{t}(0)\right)=\left(u_{0}, v_{0}\right)
$$

with the regularity : $\forall t \geq 0, \quad\left(u(t), u_{t}(t)\right) \in D(A) \times V$, and for all $T>0,\left(u, u_{t}\right)$ belongs to $W^{1, \infty}((0, T) ; V \times H)$.

(ii) For all $\left(u_{0}, v_{0}\right) \in V \times H$, there exists a unique weak solution $u=(y, \xi)$ of (3.2) such that $\left(u, u_{t}\right)$ belongs to $\mathcal{C}^{0}((0,+\infty) ; V \times H)$.

Remark. Note that, for strong solutions, $\xi_{t}(\cdot)=y_{t}(1, \cdot)$.

Proof. If we assume (2.2), it is a direct application of Theorem 1. Using an a priori estimate, we can prove that it is sufficient to assume (3.3) instead of (2.2). Indeed the energy inequality gives

$$
\forall t>0, \quad E(t) \leq E(0) .
$$

In particular,

$$
\forall t>0, \quad \xi_{t}(t)^{2} \leq 2 E(0)
$$

Set $A=\sqrt{2 E(0)}$. Since $\xi_{t}(t)$ belongs to $[-A, A]$ for all $t \in \mathbb{R}_{+}$, we can assume that $\lambda \mapsto q(\lambda)+m_{A} \lambda$ is increasing on $\mathbb{R}$ (we modify $q$ on $\mathbb{R} \backslash[-A, A]$ if necessary).

\subsection{Weak stabilization}

Applying Theorem 2, we obtain weak stabilization (with no assumption of monotonicity at all).

Corollary 2. Assume (3.3) and (2.3)-(2.5). For all $\left(u_{0}, v_{0}\right) \in V \times H$, the solution $u=(y, \xi)$ of $(3.2)$ satisfies

$$
\left(y(t), y_{t}(t)\right) \underset{t \rightarrow+\infty}{\rightarrow}(0,0) \text { weakly in } H^{1}(0,1) \times L^{2}(0,1),
$$

and

$$
\left(\xi(t), \xi_{t}(t)\right) \underset{t \rightarrow+\infty}{\rightarrow}(0,0) .
$$

Proof. It is a consequence of Theorem 2 since (2.5) is verified, i.e.

$$
\left\{\begin{array}{l}
y \in H^{1}(0,1), y(0)=0, y(1)=\xi \\
-y_{x x}=\omega^{2} y, 0<x<1, \\
y_{x}(1)=\omega^{2} y(1), \\
\underline{\text { and } \xi=0,}
\end{array} \quad \Rightarrow y \equiv 0 .\right.
$$




\subsection{Strong stabilization}

Finally, adding an assumption of local monotonicity on $q$, we deduce strong stabilization.

Corollary 3. Assume (3.3) and (2.3)-(2.5). Assume also that there exists $\delta>0$ such that $q$ is increasing on $[-\delta, \delta]$. Then for all $\left(u_{0}, v_{0}\right) \in V \times H$, the solution $u=(y, \xi)$ of (3.2) satisfies $E(t) \underset{t \rightarrow+\infty}{\rightarrow} 0$.

Proof. Weak stabilization implies in particular

$$
\xi_{t}(t) \rightarrow 0, \text { as } t \rightarrow+\infty
$$

There exists $t_{0}>0$ such that

$$
\forall t \geq t_{0}, \quad \xi_{t}(t) \in[-\delta, \delta]
$$

Let $\bar{q}: \mathbb{R} \rightarrow \mathbb{R}$ the continuous increasing and bounded function defined by :

$$
\begin{cases}\bar{q}(\lambda)=q(\lambda) & \text { for } \lambda \in[-\delta, \delta], \\ \bar{q}(\lambda)=q(\delta) & \text { for } \lambda \geq \delta, \\ \bar{q}(\lambda)=q(-\delta) & \text { for } \lambda \leq-\delta .\end{cases}
$$

Let $u$ be the solution of (3.2). We introduce the solution $\bar{u}$ of the equation obtained from (3.2) by changing $q$ by $\bar{q}$ and $\left(u_{0}, v_{0}\right)$ by $\left(\bar{u}\left(t_{0}\right), \bar{u}_{t}\left(t_{0}\right)\right)$. This problem is wellposed. Since $q$ equals $\bar{q}$ on $[-\delta, \delta]$, we deduce

$$
\forall t \geq 0, \quad\left(u\left(t+t_{0}\right), u_{t}\left(t+t_{0}\right)\right)=\left(\bar{u}(t), \bar{u}_{t}(t)\right) .
$$

Thus the problem reduces to prove strong stabilization of $\left(\bar{u}(t), \bar{u}_{t}(t)\right)$. Since $\bar{q}$ is increasing and bounded, strong compactness of trajectories holds and strong stabilization follows from classical theory, using the invariance principle of LaSalle (for example, we can apply the abstract result of [2]).

\subsection{Comments and other applications}

The method used to prove 3 also applies to other hybrid systems. We refer to [12] for other examples. In particular, it applies to the system studied by E. Feireisl and O. Dowd in [5]. For a model of an overhead crane consisting of a cable carrying a load mass, and under a hypothesis of local monotonicity, they proved strong stabilization of all strong solutions. The strategy was the same except the main step. Indeed, they proved that $\xi(t)=y_{t}(1, t) \rightarrow 0$ as $t \rightarrow+\infty$ with direct estimates (that required regularity of the solution). Thus they obtained strong stabilization only for strong solutions (due to the lack of contraction of the semigroup associated to the equation, they could not deduce strong stabilization for weak solutions). Here we also proved strong stabilization for weak solutions. Indeed the main step $\xi(t) \rightarrow 0$ as $t \rightarrow+\infty$ is obtained as a direct consequence of the general result of weak stabilization. Thus it is still true for weak solutions (since, for weak stabilization, the estimate that we need is just the boundedness of the solution in the energy space which does not 
require more regularity on the solution). We also refer to [4] for an other result of weak stabilization that applies to hybrid systems and from which strong stabilization can be deduced.

Thanks. The author is grateful to M. Pierre for a lot of instructive discussions on this subject, to B. Rao for suggesting to apply these results of weak stabilization to hybrid systems and to F. Conrad for his remarks on this paper.

\section{References}

[1] H. Brezis, Opérateurs maximaux monotones et semi-groupes de contraction dans les espaces de Hilbert, North-Holland, Amsterdam, 1973.

[2] F. Conrad, M. Pierre, Stabilization of second order evolution equations by unbounded nonlinear feedbacks, Ann. Inst. H. Poincaré Anal. Non Linéaire 11 (1994), 5, 485-515.

[3] C.M. Dafermos, Asymptotic behavior of solutions of evolution equations, in "Nonlinear Evolution Equations" (M.G. Crandall, ed.), Academic Press, NewYork, 1978, 103-123.

[4] G. O'Dowd, Stabilisation d'équations d'évolution par un contrôle non monotone: la méthode du problème factice, in progress.

[5] E. Feireisl, G. O'Dowd, Stabilisation d'un système hybride par un feedback non linéaire, non monotone, C. R. Acad. Sci. Paris Sér. I 326 (1998), 323-327.

[6] A. Haraux, Stabilization of trajectories for some weakly damped hyperbolic equations, J. Differential Equations 59 (1985), 2, 145-154.

[7] A. Haraux, Remarks on weak stabilization of semilinear wave equation, to appear.

[8] O. Morgul, B. Rao, F. Conrad, On the stabilization of a cable with a tip mass, IEEE Trans. Automatic Control 39 (1994), 10, 2140-2145.

[9] M. Pierre, J. Vancostenoble, Strong decay for one-dimensional wave equations with nonlinear nonmonotone boundary feedbacks, in progress.

[10] M. Slemrod, Weak asymptotic decay via a "relaxed invariance principle" for a wave equation with nonlinear, nonmonotone damping, Proc. Roy. Soc. Edinburgh 113A (1989), 87-97.

[11] J. Vancostenoble, Stabilisation faible de l'équation des ondes par un contrôle non linéaire, non monotone, Inst. Élie Cartan, Univ. Nancy I (1997), 3.

[12] J. Vancostenoble, Stabilisation non monotone de systèmes vibrants et contrôlabilité, Ph. D. Thesis, Univ. Rennes I, 1998.

[13] J. Vancostenoble, Weak asymptotic stability of second order evolution equations by nonlinear and nonmonotone feddbacks, SIAM J. Math. Anal. 30, 1 (1998), 140-154. 
[14] J. Vancostenoble, Weak asymptotic decay for a wave equation with weak nonmonotone damping, in progress. 